\title{
Fertility with early reduction of ovarian reserve: the last straw that breaks the Camel's back
}

\author{
Sabahat Rasool ${ }^{1 *}$ (I) and Duru Shah ${ }^{2}$
}

\begin{abstract}
Diminished fertility and poor ovarian response pose a conundrum to the experts in the field of reproductive medicine. There is limited knowledge about the risk factors of diminished ovarian reserve other than the iatrogenic ones. One of the leading causes of infertility in women today is diminished ovarian reserve (DOR). DOR is characterized by a low number of eggs in a woman's ovaries and/or with poor quality of the remaining eggs, which boils down to impaired development of the existing eggs, even with assisted reproductive techniques. A good number of such women with low ovarian reserve may conceive with their own eggs, if they are given individualized treatment that is tailored for their profile. Such patients should be counseled appropriately for an aggressive approach towards achieving fertility. The sooner the treatment is started, the better the chances of pregnancy.
\end{abstract}

Keywords: Ovarian reserve, Diminished ovarian reserve, Reduced fertility, Poor ovarian response, Poor responders

\section{Background}

Little is known about the risks, and management of diminished ovarian reserve (DOR), which is affected by age, genetics and environmental factors. The one thing certain about DOR is that it is irreversible and that these women are at risk of poor ovarian response to ovarian stimulation in Assisted Reproductive Technologies (ART) $[1,2]$. DOR is a poor prognostic factor in ART, because of a decline in the quantity and quality of oocyte [3]. Age is the most well - known contributing factor to DOR, and probably the most important prognostic factor in fertility treatment of women with DOR [3].Age-related abnormal vascularization, oxidative stress, free radical imbalance, toxic and genetic changes, all contribute to the declining oocyte quality, which translates into abnormal fertilization, and disordered embryo implantation [3]. DOR is associated with poor ovarian response to ovarian stimulation, higher cycle cancellation rates and lower pregnancy rates during In Vitro Fertilization (IVF) [4].

\footnotetext{
* Correspondence: sabahatrasool@yahoo.co.in

'Gynaecworld, Kwality House, 1st Floor, Kemps Corner, Mumbai, India

Full list of author information is available at the end of the article
}

\section{Definition}

DOR is defined as a decrease in the number of quality \& quantity of oocytes [5]. It is used to describe women of reproductive age with regular cycles mostly ovulatory, whose response to stimulation or fecundity is reduced compared to women of comparable age. It is distinct from menopause or premature ovarian insufficiency. A woman's chronological age is not the only determinant of ovarian reserve. Some studies have demonstrated an association between the cause of DOR and IVF outcome.

Poor ovarian response (POR) implies a subnormal follicular response, which means less number of eggs retrieved after ovarian stimulation during IVF [4]. ESHRE defined POR using Bologna criteria in order to standardize the definition, since the variability in the definition of POR was striking. According to Bologna criteria, POR is defined as "when at least two of the following three characteristics are present":

1. Advanced maternal age $>40$ yrs. or any other risk factors for poor ovarian response.

2. Previous POR ( $\leq 3$ oocytes with conventional stimulation of $>149$ IU FSH daily), and 
3. An abnormal ovarian reserve test $(\mathrm{AFC}<5-7$, or $\mathrm{AMH}<0.5-1.1 \mathrm{ng} / \mathrm{ml})$

Two episodes of POR after maximal ovarian stimulation are sufficient to label a patient as a poor responder [6]. The most important reason for a poor ovarian response is DOR. Some subgroups of DOR, based on the cause of DOR, show better IVF outcome than others. A recent retrospective study showed better response to IVF in DOR caused by surgery for endometrioma compared to idiopathic group [7].

Yun $\mathrm{H}$, et al. studied the IVF outcome of women with diminished ovarian reserve in 99 cycles and reported a clinical rate of $11.5 \%$ per cycle, cancellation rate of $34.4 \%$. They found that DOR caused by previous ovarian surgery had better pregnancy outcome, while that caused by chemotherapy / gonadotoxic therapy had significantly higher cycle cancellation rate [4]. Studying the trends in DOR assignment in the Society for Assisted Reproductive Technology (SART) Clinic outcomes Reporting System Database and evaluating its accuracy for POR prediction, Devine K, et al. reported an increased prevalence of DOR from $19 \%$ to $26 \%$ from 2004 to 2011 among 181,536 cycles studied [8]. The incidence of POR decreased from $32 \%$ to $30 \%$ among cycles clinically assigned as DOR. Basal FSH $\geq 12 \mathrm{v} / \mathrm{s}$ clinical management assignment of DOR had a higher specificity (92.2\% v/s 81.6\%) and positive predictive value $(38.3 \% \mathrm{v} / \mathrm{s} 30.9 \%)$ for predicting POR. They concluded that despite increasing DOR prevalence, the ability of clinical DOR to predict POR in the concurrent cycle worsened. More accurate markers of POR are needed to minimize patient anxiety, under and over diagnosis of POR. Possible explanations for increased DOR prevalence include advanced age, more diagnostic modalities and to explain suboptimal success rates to the patients labeled such.

\section{Causes of DOR [9-11]:}

Idiopathic

Chemotherapy

Radiotherapy

Genetic mutations like FMR

Smoking

Ovarian surgeries

Autoimmune

Mumps

Galactosemia

Tubal surgery

Idiopathic

Chemotherapy depletes primordial follicles in a dose and drug-dependent manner. Risk of toxicity during chemotherapy increases with age. Similarly, radiation affects ovaries depending on the dose, field and age of the patient [12]. Other causes of DOR include iatrogenic ovarian surgeries, uterine artery ligation, laparoscopic salpingectomy, genetic diseases (Turner's galatosemia, Fragile X, FSH receptor and Inhibin B mutations), enzyme defects, mumps oophorihis, autoimmunity (Polyglandular syndrome, lymphocytic oophoritis, Addison's disease, Hashimoto thyroiditis, celiac disease) and metabolic (Galactosemia)[13].

Idiopathic diminished ovarian reserve involves accelerated oocyte apoptosis. According to Barkers hypothesis, maternal endocrine disturbance during in utero life may result in DOR in the female fetus [14].

\section{Ovarian reserve estimation}

Ovarian reserve is used to describe a woman's reproductive potential by means of the quality and quantity of the oocytes her ovaries possess [15]. Ovarian reserve tests (ORT) aim at identifying women at risk of hypo or hyper response to ovarian stimulation, detecting reproductive lifespan and approximate menopausal timing and counseling and planning about a family, and individualizing management to optimize ovarian response whilst minimizing risks. Nevertheless, it is of utmost importance to understand that ORTs should not be used as a sole criterion to deny ART or other treatments to any patients. If ORTs indicate a diminished ovarian reserve, it means that pregnancy is less likely but not impossible. Though we use a battery of ORTs as a proxy for oocyte number, testing the oocyte quality or competence using these tests is poor.

Ideal ORT needs to be affordable, non-invasive, sensitive and specific, with minimal inter and intra cycle variability, with good sensitivity to detect the decline in ovarian reserve at a stage where timely interventions could lead to pregnancy [16]. ORTs should be offered to women at risk of DOR, not to the low-risk population.

ORT that are used as of now are biochemical, provocative and sonographic imaging of ovaries. The tests used for assessing ovarian reserve include basal day -3 follicle stimulating hormone (FSH, introduced in 1998). Clomiphene citrate challenge test (CCCT, 1989), gonadotropins releasing - hormone agonist stimulation test (GAST, 1989), Inhibin -B (1997), antral follicle count (AFC, 1997) and antimullerian hormone (AMH, 2002.)

The provocative tests like CCCT and GnRH-agonist stimulation test are literally out of practice now and FSH, AFC and AMH are being used extensively $[17,18]$.

\section{Basal FSH}

This test is based on the negative feedback of FSHpituitary secretion by ovarian factors like estradiol and inhibin B. Normal women have adequate 
quantities of ovarian hormones in early follicular phase, which maintains the FSH levels with normal range. However, women with depleting oocyte pool and diminished ovarian reserve will present with elevated basal FSH levels. Though basal FSH levels have significant inter and intra cycle variability, but when combined with basal estradiol, the sensitivity and specificity of basal FSH to test ovarian reserve is accentuated [19]. The specificity for using FSH to predict poor ovarian response is $45-100 \%$ ( $\leq 4$ retrieved occytes). However the sensitivity is low $11-86 \%$ [19, 20]. FSH cut off values have been reset from 25 IU to $10 \mathrm{IU}$ to detect expected poor ovarian response and failure to conceive [19].

\section{Basal estradiol}

Early follicular estradiol $\left(E_{2}\right)$ levels are better used with basal FSH to assess ovarian reserve. Basal $E_{2}$ has poor inter and intra cycle reliability. High basal $E_{2}$ with normal FSH levels $(>60-80 \mathrm{pg} / \mathrm{ml})$ may indicate poor response and increased cycle cancellation rates [21].

\section{Antimullerian hormone (AMH)}

$\mathrm{AMH}$, a glycoprotein of the transforming growth factor - B (TGF - B), is produced by granulosa cells of small and large preantral and small antral follicles [22]. AMH levels peak at 25 years of age, gradually declining thereafter and reaching undetectable levels a few years before menopause [23-25]. AMH is secreted during early follicular stage by follicles up to $6 \mathrm{~mm}$ in size, is relatively gonadotropin-independent, and remain relatively constant within and between menstrual cycles [26-30]. Good evidence suggests that if significant fluctuations in $\mathrm{AMH}$ do occur, they are limited to younger women [31],, women with high AMH and aged women with low ovarian reserve [31, 32]. AMH is one of the best tests for ovarian reserve. It correlates very well with primordial follicle pool, ovarian response during ovarian stimulation and reliably predicts menopausal timing [33, 34]. $\mathrm{AMH}$ declines years before a rise in FSH is seen, thus proves to be a more sensitive biomarker of ovarian reserve $[35,36]$. A systematic review of studies on women undergoing ovarian stimulation with gonadotropins by La Marca et al. (2010) identified low AMH cut-off points of $0.1-1.66 \mathrm{ng} / \mathrm{mL}$ to have sensitivities of $44-97 \%$ and specificities between 41 and $100 \%$ for predicting a poor ovarian response [22]. In addition, AMH correlates very strongly with the risk of ovarian hyper stimulation to gonadotropins, sensitivities range from 53 to $90.5 \%$ and specificities range from 70 to $94.4 \%$ using a cut - off of $3.36-5.0 \mathrm{ng} / \mathrm{mL}[22]$. AMH is, however, a poor test for prediction of pregnancy and live births after ART, as is clearly concluded in 2 recent meta-analyses by Iliodromoti et al. [37] and Tal R, et al. [38] AMH should not be the sole criterion for denying fertility treatment. On the contrary, such women should be thoroughly counseled about possible low oocyte yield, high cycle cancellations and poor embryo quality.

AMH assessment is done using Enzyme -linked immunosorbent assay (ELISA) Gen II, Beckman Coulter Inc.; Brea, CA [39]. Newer automated AMH assay platforms are reported to offer better precision and sensitivity, faster results when compared to standard ELISA assays $[40,41]$. Certain factors tend to influence the AMH values. These include PCOS (high), ovarian suppression with oral contraceptive pill (OCP) or GnRH agonists (Low), race and ethnicity, ovarian surgery, current smoking (low), and low vitamin D (low) [42-47]. Women with polycystic ovary morphology had significantly higher AMH levels than women in the control group. The prevalence of PCOS increased from $21 \%$ in the low-AMH $(<4 \mathrm{ng} / \mathrm{mL})$ group to $37 \%$ in the moderate-AMH (4-11 ng/mL) group and $80 \%$ in the highAMH (>11 ng/mL) group [42]. Kallio $\mathrm{S}$ et al. studied the effects of oral contraceptives on serum levels of $\mathrm{AMH}$ and other hormones and concluded that antimullerian hormone $(\mathrm{AMH}), \mathrm{FSH}$, inhibin $\mathrm{B}, \mathrm{LH}$, and E2 levels had decreased significantly after 9 weeks of treatment [43].

Waylen AL et al. studies the effects of cigarette smoking on reproductive hormones (follicular serum concentrations of inhibin B hormone, FSH and $\mathrm{AMH}$ ) in women of reproductive age and found a trend towards lower AMH and FSH concentrations in smokers, though it did not reach statistical significance [46]. A correlative and intervention study by Dennis NA, et al. to determine whether serum levels of AMH correlated with 25hydroxyitamin D [25(OH)D)] status, and concluded that the change in AMH levels correlated with the magnitude of change in vitamin D levels and that cholecalciferol supplementation prevented seasonal AMH change [47].

\section{Antral follicle count (AFC)}

AFC is the sum of follicles in both ovaries seen on ultrasound imaging during early follicular phase (Day 2-4). Antral follicles are defined as those measuring between 2 and $10 \mathrm{~mm}$ on a 2-dimensional plane. AFC is easy and quick to carry out, with good inter cycle and inter observer reliability [20], but has a compromised precision in women with weight extremes [20] and is dependent on the time of the cycle. A systematic review of ovarian reserve tests and IVF outcome by Broekman FJ et al. [20] concluded that low AFC is associated with poor ovarian response but has low predictability for pregnancy. The specificity of AFC for non- pregnancy predictor ranges between 64 and $98 \%$ but sensitivity stays low at $7-34 \%$ [20]. AFC also overestimates the actual number of FSH- 
sensitive follicles and oocyte yield, as it also measures atretic follicles of the same size [48].

\section{Ovarian volume and blood flow}

Ovarian volume, calculated as $\mathrm{D}_{1} \times \mathrm{D}_{2} \times \mathrm{D}_{3} \times 0.52$ by measuring each ovary in three planes. This test has a limited value in testing ovarian reserve as it does not correlate well with pregnancy prediction [49]. In a metaanalysis where ovarian blood flow assessment was studies to predict IVF outcomes, it was concluded that this test is of unclear value [50].

\section{Which one is better - The debate}

Leader B, et al. studied 5354 women to examine discordance between $\mathrm{AMH}$ and FSH results and found 1 in 5 women with discordant $\mathrm{AMH}$ and FSH values defined as $\mathrm{AMH}<0.8 \mathrm{ng} / \mathrm{ml}$ (concerning) with FSH $<10 \mathrm{IU} 0 \mathrm{~L}$ (reassuring) or $\mathrm{AMH}>0.8 \mathrm{ng} / \mathrm{ml}$ (reassuring) with $\mathrm{FSH} \geq 10 \mathrm{IU} / \mathrm{L}$ (concerning). AMH is more sensitive than FSH in diagnosing DOR, as was found in the study [51]. When ovarian reserve tests are discordant, it's safe to go with an intermediate value between the two and administer an intermediate dose of gonadotropins stimulation [16] (Table 1).

AMH $<0.5 \mathrm{ng} / \mathrm{ml}$ predicts an oocyte yield of $\leq 4$ oocytes [22]. Patient should be counseled about an aggressive fertility treatment using microdose GnRH agonist flare with high starting gonadotropin dose or late luteal estradiol priming with or without late luteal presuppression antagonist with high starting gonadotropins dose $[16,22,52]$.

AMH $\geq 1.0 \mathrm{ng} / \mathrm{ml}$ but $\leq 3.5 \mathrm{ng} / \mathrm{ml}$ predicts normal ovarian response and such women should undergo conventional GnRH agonist pr antagonist protocols [53-56].

\section{Whom to test?}

Since nearly $10 \%$ of females undergo accelerated oocyte pool depletion, which leads to sub fertility and infertility early on, and with the present scenario of many women delaying childbearing, this issue may be of concern since it means many more women will end up in a poor ovarian response [57]. In the West, $25 \%$ women do not attempt pregnancy till 35 years of age [58]. Azhar E, et al. concluded that the knowledge of ovarian reserve would lead women to modify their reproductive decisions and make alternative decisions [59]. Women may become more well-versed with their reproductive lifespan and menopausal timing if offered ovarian reserve tests. Young cancer patients, who are planning to undergo gonadotoxic therapies, may especially benefit by ORTs.

Though there is a hot debate between the opponents and proponents of ovarian reserve testing in the general population, some advantages it offers includes identifying younger women with DOR who have a higher risk of early loss of fertility potential and advising them about their limited fertility option accordingly $[60,61]$.

\section{Preventing iatrogenic diminished ovarian reserve}

Atabekoglu $\mathrm{C}$ et al. found that abdominal hysterectomy causes $30 \%$ more loss of ovarian function, although not statistically significant [62]. Hysterectomy was found to lead to early menopause and hysterectomy with unilateral oophorectomy to even earlier menopause in a prospective cohort study [63].

In a meta-analysis where the effect of surgery for endometriomas on ovarian reserve was studied, it was concluded that there's a significant decline in AMH levels after ovarian cystectomy, with a mean weighted difference of $1.13 \mathrm{ng} / \mathrm{ml}$. Endometriotic cyst excision was found to have a negative impact on ovarian reserve in terms of reduced AMH levels [64].

A recently published meta-analysis on changes in $\mathrm{AMH}$ levels after laparoscopic ovarian drilling (LOD) concluded that LOD markedly reduces AMH levels with PCOS who have normal or low AMH levels [65]. Salpingectomy was not shown to compromise ovarian reserve in the short- term.

Thus avoiding unnecessary surgeries and offering alternative therapies wherever indicated, will help in reducing the burden of iatrogenic diminished ovarian reserve.

In a recent meta-analysis by Mohamed AA et al., the mean age of menopause in current smokers is $1.74 \pm 0.46$ years earlier than non-smokers [66]. However, a dose-dependent effect was negligible [67].

Among young cancer patients, fertility preservation may be done by ovarian tissue cryopreservation (OTC), oocyte or embryo freezing prior to chemo/radiotherapy. Gonadotropin-releasing hormone analogue $(\mathrm{GnRH}-\mathrm{a})$

Table 1 [16] Comparison between FSH, AMH and AFC

\begin{tabular}{llll}
\hline TEST & BASAL FSH & AFC & AMH \\
\hline Cut-offs used for sensitivity and specificity & $10-20$ & $<3-4$ follicles, total & $0.1-1.66(\mathrm{ng} / \mathrm{ml})$ \\
Sensitivity for Poor response (\%) & $11-86$ & $9-73$ & $44-97$ \\
Specificity for Poor response (\%) & $45-100$ & $73-97$ & $41-100$ \\
Sensitivity for Non Pregnancy (\%) & $3-65$ & $7-34$ & $19-66$ \\
Specificity for Non Pregnancy (\%) & $50-100$ & $64-98$ & $55-89$ \\
\hline
\end{tabular}


use has been found promising in preserving fertility if administered during chemotherapy [68]. Prior to pelvic radiotherapy, ovarian transposition can be done especially for pre-pubertal children.

Hormonal therapy for well-differentiated endometrial cancer and radical trachelectomy in early stage cervical cancer among young women may help preserve fertility to a great extent [69].

So far as the iatrogenic causes of DOR are concerned, strategies can be made to prevent damage to ovarian reserve. However, once ovarian reserve diminishes, unfortunately, not much can be done to replenish it.

\section{Social egg freezing- race against time or smoke and mirrors?}

Human eggs have a definite decline in quality as well as quantity after 30 years of age, which plummets after 35 . Social egg freezing is seen an insurance against agerelated fertility decline. Social, personal, educational and financial pressures often lead women to delay starting a family until the late thirties, by which time the chance of getting pregnant is compromised by low fecundity rates and an increased risk of miscarriage if they become pregnant. In an internet-based survey on knowledge and attitudes of women towards social egg freezing in UK and Denmark, it was found that $83 \%$ women were aware about egg freezing option and $89 \%$ considered it acceptable for social reasons [70]. Characteristics significantly limited to the intention of egg freezing included being single, age under 35 years, childlessness and a history of infertility. The efficacy of the procedure was more important than its potential side effects and cost.

\section{The problem of poor ovarian response}

Poor responders are a heterogeneous population as far as the pregnancy is concerned. Age and oocyte yield have a substantial effect on the pregnancy prospects in such patients [70]. The existing studies and research do not offer any clear - cut guidelines for clinical handling. A comprehensive review of trails published on "poor responders" by Papathanassiou A, et al. [71] concluded that a variety of definitions were used to define poor responders and a battery of interventions were done for head-to-head comparisons. This led to glaring discrepancies in the results of trials [71]. The existing criteria and definitions have not been able to sub classify POR patients who could benefit from particular interventions.

Comparing the various interventions for poor responders in IVF, it was concluded that the evidence to routinely support any particular intervention, either for pituitary down regulation, ovarian stimulation or use of adjutants, is insufficient [72]. The debate of oocyte quantity versus quality tips in favour of quality. Blastocyst euploidy rates drop drastically from $60 \%$ in women $<35$ years of age to $30 \%$ or lower in women aged 40-42 years, while age-related aneuploidy drastically rises [73].

Recently, the POSEIDON group (Patient - Oriented Strategies Encompassing IndividualizeD Oocyte Number) proposed a new classification of ART in patients with DOR or unexpected inappropriate ovarian response and four subgroups have been proposed based on quantitative as well as qualitative parameters which include

1. Age and expected anomaly rate,

2. Ovarian biomarkers like AFC and AMH, and

3. Ovarian response - if an earlier stimulation was performed [74].

\begin{tabular}{|c|c|}
\hline POSEIDON GROUP 1 & POSEIDON GROUP 2 \\
\hline $\begin{array}{l}\text { Young patients }<35 \text { years with } \\
\text { adequate ovarian reserve (AFC } \geq 5 \text {; } \\
\text { AMH } \geq 1.2 \mathrm{ng} / \mathrm{ml} \text { ) and with an } \\
\text { unexpected poor or suboptimal } \\
\text { ovarian response. }\end{array}$ & $\begin{array}{l}\text { Older patients }>35 \text { years with } \\
\text { adequate ovarian reserve }(\mathrm{AFC} \geq 5 \text {; } \\
\mathrm{AMH} \geq 1.2 \mathrm{ng} / \mathrm{ml} \text { ) and with an } \\
\text { unexpected poor or suboptimal } \\
\text { ovarian response. }\end{array}$ \\
\hline $\begin{array}{l}\text { - Sub group 1a: }<4 \text { oocytes* }^{*} \\
\text { - Sub group 1b: } 4-9 \text { oocytes* }\end{array}$ & $\begin{array}{l}\text { - Sub group 2a: < } 4 \text { oocytes } \\
\text { retrieved* } \\
\text { - Sub group 2b: 4-9 oocytes } \\
\text { retrieved* }\end{array}$ \\
\hline $\begin{array}{l}\text { * Retrieved after standard } \\
\text { stimulation. }\end{array}$ & $\begin{array}{l}\text { * Retrieved after standard } \\
\text { stimulation }\end{array}$ \\
\hline SEIDON GROUP 3 & POSEIDON GROUP 4 \\
\hline
\end{tabular}

Young patients $<35$ years with poor ovarian reserve pre stimulation parameters (AFC $<5$; $\mathrm{AMH}<1.2 \mathrm{ng} / \mathrm{ml})$

Older patients $\geq 35$ years with poor ovarian reserve pre - stimulation parameters (AFC $<5$; $\mathrm{AMH}<1.2 \mathrm{ng} / \mathrm{ml})$

The POSEIDON concept is expected to better classify women with low prognosis in Art, to help individualize their treatment to get at least one euploid embryo for transfer in each patient as a clear practical goal.

\section{Management}

The management of DOR \& POR can be a frustrating nightmare despite extensive studies and strategies. The strategies used are all aimed at a higher oocyte yield.

Various treatment regimens have been designed to manage the patients with diminished ovarian reserve and include high dose of gonadotropins, natural and modified natural cycles, estrogen priming, supplementation with $\mathrm{LH}$, luteal antagonists and letrozole co-treatment use of adjuncts like androgens, growth hormone, melatonin and aspirin, oocyte donation and assisted hatching. 


\section{Various protocols}

\section{High dose gonadotropins}

High dose of gonadotropins may not benefit the patient beyond a particular dose and may also increase the possibility of poor oocyte quality [75], patient discomfort and side effects. A retrospective analysis of 943 patients sub grouped according to the daily dose of gonadotropins (DD GN) was done for 1394 treatment cycles [76]. Group I received a "high dose" > 225 - 375 IU, Group II "very high dose" 376-450 IU, Group III "extremely high dose" 451-600 IU, controls received a DD GN of $\leq 225$ IU. It was found that DD and total dose of GN negatively correlated with the oocyte yield, implantation, clinical pregnancy and live birth rates $(25.9 \%, 14.6 \%, 11.4 \%$ and $4.7 \%$ in control, Groups I, II and III, respectively). Increasing the daily dose of GN higher than 450 IU or a total dose of 3000 IU / cycle is questionable. Furthermore, profound stimulation has a detrimental effect on luteal endocrine milieu and in turn affects endometrial receptivity $[77,78]$.

\section{Agonist and antagonist protocols}

Comparing 2 ovarian stimulation protocols in 440 poor responders who had all failed to become pregnant during their first IVF cycle with long GnRH agonist protocol, Merviel P, et al. found that though the group treated with a contraceptive pill + flare up GnRH agonist protocol had a higher number of embryos obtained, the implantation and ongoing pregnancy rates per transfer were the same between this group and the group with $\mathrm{GnRH}$ - antagonist protocol [79]. It was concluded that these two protocols resulted in similar implantation rates and that a customized ovarian stimulation regimen including mild ovarian stimulation, sequential IVF cycles, oocytes - embryos freeze all protocols and blastocyst transfers after screening may improve the outcome [79].

In a systematic review and meta - analysis of 12 studies, the effectiveness of GnRH antagonist in poor ovarian responders was evaluated, and it was found that stimulation period and gonadotropins dose, both were statistically significantly lower in the antagonist protocol compared to the long GnRH protocol. However, endometrial thicknesses, estrogen level on the day of HCG trigger and oocyte yield were lower in the antagonist group. The cycle cancellation and clinical pregnancy rates were not statistically significantly different between the two groups [80].

Song $\mathrm{Y}$, et al. compared the effectiveness of $\mathrm{GnRH}$ antagonist / letrozole (A/L) protocol and the microdose $\mathrm{GnRH}$ agonist flare up protocol (MF) in a systematic review and meta- analysis [81]. The clinical pregnancy rate was significantly decreased with the $\mathrm{A} / \mathrm{L}$ compared with MF protocol (RR 0.70; 95\% CI: 0.57-0.86; $p-$ $0.001)$ \& the duration of gonadotropin stimulation was lower in the A/L group. There was no significant difference in cycle cancellation rates, oocyte yield and total dose of gonadotropins. Large -scale trials are needed to assess $\mathrm{A} / \mathrm{L}$ protocol in view of the lower clinical pregnancy rate in this meta-analysis [82].

Various $\mathrm{GnRH}$ analogue protocols have been tried and tested in women with DOR. Late luteal GnRH agonist protocol may lead to excessive ovarian suppression in patients with DOR, which is not desirable as it may lead to reduced or even absent follicular response.

Various options tried in women with poor ovarian reserve include

1. Decreasing the length of suppression by using short, ultra-short, mini and micro dose flare up regimens,

2. Stopping or lowering the GnRH agonist after pituitary suppression, and

3. Using GnRH antagonist from mid-late follicular phase.

Despite widespread use of short and ultra short flare up regimens, none of the published studies have been able to establish any significant benefit on the clinical outcome in women with diminished ovarian response [81, 83, 84]. Two randomized trials failed to demonstrate any improvements in reproductive outcome when "GnRH agonist stopped protocol" was compared to the standard stimulation protocols $[85,86]$. Similarly, in a recent meta analysis, no statistically significant difference was found in clinical pregnancy rates, per cycle, duration of stimulation and total gonadotropin dose used, randomized between the GnRH agonist stopped protocol and the standard agonist protocol [87].

GnRH antagonist use is more patient friendly and decreases the amount and the number of days of gonadotropin stimulation. The introduction of $\mathrm{GnRH}$ antagonists is the key to development of milder and minimum stimulation protocols which allow for the initiation of the IVF treatment cycle in a normal menstrual cycle with an undisturbed recruitment of a cohort of follicles during the early follicular phase. This approach enables the endogenous inter-cycle FSH rise to be utilized rather than suppressed, resulting in a reduction of gonadotropins required. The treatment cycles are thus shorter and not associated with hypoestrogenic side effects related to GnRH agonist down-regulation, and reduce cancelation rates. Among the various advantages of using a $\mathrm{GnRH}$ antagonist in poor responders is the use of a newer long - acting hybrid gonadotropin, corifollitropin alfa that supports the follicle cohort for upto seven days. The corifollitropin rapidly increases serum $\mathrm{FSH}$, which results in a significantly higher exposure of 
small antral follicles to high levels of FSH during early follicular phase and reduces the burden of daily injection [88] . Promising results were reported in a retrospective study on young poor responders using a combination of corifollitropin alfa with hp.-HMG in a GnRH antagonist protocol [89].

\section{Letrozole and clomiphene citrate co-treatment}

In a recent retrospective analysis of 220 poor responders, the effects of different doses of letrozole (LZ) combined with gonadotropins (Gn) and high-dose gonadotropin stimulation in antagonist cycles were compared. Patients were divided into Group 1 (LZ $5 \mathrm{mg}$ for 5 days sequentially overlapping with Gn cycles), group 2 (LZ $7.5 \mathrm{mg}$ for 3 days sequentially with $\mathrm{Gn}$ cycles), and group 3 (highdose Gn cycles) [90]. The amount of gonadotropin used in letrozole groups was lower with comparable pregnancy and live birth rates between the groups [90].

Group 1 had significantly higher early LH elevation rate on the HCG day than groups 2 and 3. Group 2 resulted in fewer improper LH surges and better outcomes than group 1.

Standard high dose gonadotropin - antagonist protocol ( $\geq 300 \mathrm{IU} /$ day) was compared with a minimal stimulation protocol (150 IU / day) using overlapping with letrozole in antagonist cycle in poor responders, in a retrospective cohort study. The low dose protocol consisted of low dose $2.5 \mathrm{mg}$ PO over 5 days, starting from cycle day 2 . On day 4 of the cycle (day 3 of the letrozole treatment) overlapping low dose gonadotropins was started. The high-dose group received high levels of gonadotropins ( $\geq 300 \mathrm{IU} /$ day) starting from day 2 of their cycle and throughout their short antagonist cycle. Clinical pregnancy rate was found to be significantly higher in the minimal stimulation group. In conclusion, the minimal stimulation IVF protocol was less expensive and resulted in a higher clinical pregnancy and live birth rate [91]. Since one protocol being compared involved letrozole and the other did not, it is not certain that the results were not related to the use of letrozole rather than high versus low dose gonadotropins.

\section{Minimal stimulation}

A recent multi-center randomized non-infertility trial studied the pregnancy rates in mild ovarian stimulation strategy in women with poor ovarian reserve compared to those with conventional ovarian stimulation. Low dose 150 IU FSH with antagonist was compared with high dose 450 IU HMG with long mid-luteal GnRHagonist. The ongoing pregnancy rate in the minimal stimulation was $12.8 \%$ versus $13.6 \%$ for conventional stimulation group. The duration of ovarian stimulation and amount of gonadotropins used was significantly lower in the former group. The study concluded that high dosages of gonadotropins are not necessary in women with poor ovarian reserve [92].

A meta- analysis evaluated the efficiency of mild ovarian stimulation with and pregnancy outcome in poor ovarian responders and indicated that there was no significant difference for live birth and clinical pregnancy rates between conventional protocol of agonist and mild protocol of gonadotropins with $\mathrm{CC}$ and $\mathrm{GnRH}$ antagonist. It suggested that mild stimulation protocol with $\mathrm{CC}$ may give similar pregnancy outcomes in POR patients compared to the conventional stimulation protocol [93]. Oktem et al. found that severe poor responders who have previously failed to respond to microdose or antagonist protocol may benefit from CC plus HMG/Antagonist [94].

\section{The use of adjuvants}

\section{Androgen (Dehydroepiandrosterone or testosterone)}

Among the various adjuncts used to better the IVF outcome in poor responders are dehydroepiandrosterone (DHEA) and testosterone (T). DHEA and T are steroid hormones meant to increase conception rates by positively affecting follicular response to gonadotropin stimulation, in turn leading to better oocyte yield and pregnancy. Androgen also increases FSH receptor expression in granulose cells. Cochrane Database Systematic Review in 2015 indicated that pre-treatment with DHEA led to higher live birth and ongoing pregnancy rates compared to no treatment or placebo with an odds ratio of 1.88 and $95 \% \mathrm{CI}$. There was no evidence of a difference in miscarriage rates. Similarly, $\mathrm{T}$ compared to no / placebo treatment was associated with higher live birth rates with an odds ratio of 2.60 , and $95 \% \mathrm{CI}$ and no difference in the miscarriage rates. The authors concluded that in poor responders, pre-treatment with DHEA or T may improve live birth rates. In women with a $12 \%$ chance of live birth/ongoing pregnancy with placebo or no treatment, the live birth/ongoing pregnancy rate in women using DHEA was between $15 \%$ and $26 \%$. In women with an $8 \%$ chance of live birth with placebo or no treatment, the live birth rate in women using testosterone was between $10 \%$ and $32 \%$. To conclude, in poor responders undergoing ART, pre-treatment with DHEA or testosterone may be associated with improved live birth rates. The quality of evidence was moderate [95].

\section{Estradiol priming in luteal phase}

Estradiol priming in the luteal phase with or without the simultaneous use of $\mathrm{GnRH}$ antagonist was found to decrease the risk of cycle cancellation and increase the chances of clinical pregnancy in a meta-analysis of 8 studies [96]. Luteal estradiol priming improves follicle synchronization. However, further studies are needed to establish the role of estradiol priming. 


\section{Recombinant LH}

Both $\mathrm{LH}$ and FSH are required for adequate ovarian estrogen biosynthesis and folliculogenesis. Theca cell derived androgen production controlled by $\mathrm{LH}$ is necessary for estrogen production by granulose cells. In mid-to-late follicular phase, FSH induces LH/hCG receptor expression in granulosa cells of large follicles [97]. In a recent meta-analysis of 40 studies, significantly more oocytes were retrieved and significantly higher clinical pregnancy rates were observed with recombinant - human FSH plus recombinant $\mathrm{LH}$ versus recombinant-FSH treatment alone in poor responders, suggesting that there's an increase in the clinical pregnancy rates of $30 \%$ in poor responders with the addition of recombinant $\mathrm{LH}$.

\section{Growth hormone (GH)}

The use of $\mathrm{GH}$ is believed to modulate the action of FSH on granulose cells by up regulating the local synthesis of Insulin - like growth factor - I (IGF -1) [98]. Cycle cancellation rates and dose of gonadotropins were reduced in patients who received $\mathrm{GH}$ in a metaanalysis of 6 RCTs involving 169 patients [99]. Another meta-analysis involving $11 \mathrm{RCTs}$ with 663 patients also reported significantly higher clinical pregnancy and live birth rates, oocyte yield, MII oocytes and E2 levels on the day of human chorionic gonadotropin (HCG). These two recent meta-analyses have suggested a significant improvement in clinical pregnancy and live birth rates, oocyte yield, mature oocytes with the use of GH [99, 100]. Large scale multi centric double blinded randomized controlled trials are needed to establish the efficacy and safety of $\mathrm{GH}$ beyond doubt in poor responders.

\section{Melatonin}

Melatonin, a pineal gland hormone, regulates physiologic reproductive behavior and acts as a free radical scavenger [101]. Melatonin supplementation has been used to improve the outcome of IVF cycles in PCOS patients and women with DOR [102]. The mean estradiol level on the trigger day, mature oocyte yield and top quality embryos were higher among women who received melatonin. However, there was no difference on other ART outcomes between melatonin and no-melatonin groups [102].

\section{Aspirin}

Good intra-ovarian blood flow is believed to improve the delivery of gonadotropic hormones for folliculogenesis and poor ovarian blood flow is linked to poor reserve $[103,104]$. The use of aspirin in IVF has been a topic of debate. The conclusion of a meta-analysis in 2007 was that the clinical pregnancy rate per embryo transfer did not improve with low-dose aspirin; and the use of aspirin should not be routinely recommended [105].

\section{Oocyte accumulation}

Novel vitrification technologies are being used to accumulate oocyte from several ovarian stimulation cycles, creating a similar situation as in normorespnder. Oocyte accumulation by vitrification followed by insemination yields comparable success in low responders [106]. It was possible to achieve higher live birth rates per patient and reduce dropouts with oocyte accumulation in a study by Cobo, et al. [106].

\section{Dual stimulation/double stimulation (follicular and luteal phase)}

More recent evidence suggests that folliculogenesis occurs in a wave-like fashion through the menstrual cycle, and that there are multiple recruitment waves [107]. This has opened up a new horizon of opportunities to utilize ovarian stimulation in luteal phase following oocyte retrieval with follicular phase stimulation in the same cycle [108]. Typically, a luteal phase stimulation starts 2-7 days after oocyte retrieval in the same cycle. Either Gonadotropins or CC or Letrozole are used followed by trigger at lead follicle size of $18 \mathrm{~mm}$. Embryo freezing is recommended in view of anticipated endometrial asynchrony. A randomized open-label pilot trial studies the luteal initiation of Gonadotropins in poor responders. The number of oocytes retrieved was similar during follicular and luteal phases. Serum estradiol levels, pregnancy and live birth rates did not differ between the two stimulation phases [109] In yet another study to evaluate the efficacy of double stimulation in poor responders undergoing mild ovarian stimulation it was showed that stimulation started in luteal phase could result in retrieval of more oocytes in a short period of time, which is important in poor responders [110]. In a more recent study, it was found that mean number of oocytes retrieved, mature oocytes and zygotes with two pronuclei was significantly higher for luteal stimulation collections in double stimulation cycles. The rate of clinical pregnancy and embryo implantation increased progressively from pure follicular phase embryos to mixed embryos to pure luteal phase embryos [111]. Larger double-blinded randomized studies are needed to establish the role of double stimulation beyond doubt.

Table 2 ART cycles in patients with DOR

\begin{tabular}{lllll}
\hline Age in years & $25-30$ & $31-35$ & $36-40$ & $>40$ \\
\hline Number of cycles & 9 & 17 & 60 & 31 \\
Mean AMH ng/ml & 1.09 & 0.99 & 0.74 & 0.4 \\
Mean AFC & 4 & 3 & 2 & 1 \\
Pregnancy Rate \% & 33.33 & 29.4 & 21.82 & 12.9 \\
\hline
\end{tabular}


Table 3 Cycle Characteristics \& outcome (POSEIDON Criteria)

\begin{tabular}{|c|c|c|c|c|c|c|}
\hline & Group1a & Group 1b & Group 2a & Group 2b & Group 3 & Group 4 \\
\hline Number of patients & 8 & 18 & 12 & 25 & 11 & 27 \\
\hline Age in years & $<35$ & $<35$ & $>35$ & $>35$ & $<35$ & $\geq 35$ \\
\hline Mean basal FSH mIU/ml & 9.1 & 9.68 & 6.54 & 9.93 & 6.45 & 9.38 \\
\hline Mean basal E2 & 65 & 57.5 & 29 & 32.57 & 50 & 38.12 \\
\hline Mean AFC & 8 & 9 & 5 & 6 & 4 & 2 \\
\hline Mean AMH ng/ml & 3.1 & 2.82 & 1.42 & 2.19 & 0.64 & 0.71 \\
\hline Number of cycles & 10 & 22 & 12 & 28 & 13 & 32 \\
\hline Total Gn dose IU & 6675 & 7050 & 5850 & 6431 & 8250 & 8962 \\
\hline Mean number of oocytes retrieved & 3 & 5 & 3 & 6 & 4 & 3 \\
\hline Mean number of mature oocytes & 3 & 5 & 2 & 4 & 4 & 2 \\
\hline Fertilization Rate \% & 83.33 & 80 & 66.66 & 75 & 75 & 100 \\
\hline Cleavage Rate \% & 66.66 & 60 & 66.66 & 75 & 75 & 100 \\
\hline Pregnancy Rate \% & 40 & 45.45 & 25 & 39.28 & 15.38 & 37.50 \\
\hline
\end{tabular}

\section{Oocyte donation}

Egg donation may be the last efficient resort to offer hope to patients with diminished ovarian reserve and poor response. Though the pregnancy rates in women who use egg donation are at least as good as normoresponders, the decision is often difficult to make. Moreover, the facilities for egg donation, the acceptability and adequate counseling may not be available worldwide.

\section{Others}

The newer yet-to-be established approaches in poor responders include ovarian transplantation, mitochondrial transfer and stem cell based neo-oogenesis [112, 113]. Some new data support the possibility to experimentally restore fertility in women with depleted ovarian reserve. Following the findings of germ line stem cells in adult mouse ovaries, several studies are trying to attempt isolating mitotic germ cells in adult human ovaries [114]. Virant - Klun et al. [114] tried to isolate the putative ovarian stem cells (OSCs) from the ovarian surface epithelium (OSE) in women with no naturally present oocytes and follicles. OSE scraping isolated small round cells, $2-4 \mu \mathrm{m}$ in diameters, they were cultured and some oocyte like cells developed and reached $20 \mu \mathrm{m}$ diameter and were termed "embryonic-like stem cells of the adult" and these oocyte - like cells underwent parthenogenetic activation to form blastocyst - like structures [114].

Experiments are being carried out towards development of artificial gametes from diploid somatic cells. Problems being faced are the inability of the somatic cells to reduce their chromosomes with the requisite fidelity and efficacy of a germ cell, which leads to high incidence of chromosomal abnormalities resulting from non- disjunction. More research is needed to determine whether these approaches are a realistic paradigm in the management of women with no follicles / eggs.

Our experience with diminished ovarian reserve and poor ovarian response [115] (Tables 2 and 3).

\section{Conclusions}

Despite a plethora of studies on diminished ovarian reserve and poor ovarian response, we are yet to reach a consensus and conclusion about their apt definition and management. Increasing the dose of gonadotropins, luteal phase gonadotropins, flare up agonist protocols, GnRH antagonists, supplementation with $\mathrm{GH}$, Clomiphene citrate, letrozole, androgens and aspirin are among the various management strategies. We believe a thorough patient counseling and protocol personalization are the keys to optimize reproductive outcomes in such patients. Women with DOR should be appropriately counseled to undergo a rather- aggressive approach to achieve pregnancy before it is too late.

\footnotetext{
Abbreviations

AL: Antagonist letrozole; AFC: Antral follicle count; AMH: Antimullerian hormone; ART: Assisted Reproductive Technologies; CC: Clomiphene citrate; CCCT: Clomiphene citrate challenge test; Cl: Confidence interval; DD GN: Daily dose of gonadotropins; DHEA: Dehydroepiandrosterone; DOR: Diminished ovarian reserve; ELISA: Enzyme -linked immunosorbent assay; FSH: Follicle stimulating hormone; GAST: Gonadotropins releasing hormone agonist stimulation test; GH: Growth Hormone; Gn: Gonadotrpins; $\mathrm{GnRH}$ - a: Gonadotropin-releasing hormone analogue; HCG: Human chorionic gonadotropin; HMG: Human Menopausal Gonadotropin; IGF -1: Insulin - like growth factor - I; IU: International Units; IVF: In Vitro Fertilization; LH: Luteinizing hormone; LOD: Laparoscopic ovarian drilling; OCP: Oral contraceptive pill; ORT: Ovarian reserve tests; OSCs: Ovarian stem cells; OSE: Ovarian surface epithelium; OTC: Ovarian tissue cryopreservation; POR: Poor ovarian response; POSEIDON group: (Patient - Oriented Strategies Encompassing IndividualizeD Oocyte Number); SART: Society for Assisted Reproductive Technology; T: Testosterone
}

Funding

No funding. 


\section{Availability of data and materials}

N/A

\section{Authors' contributions}

Both SR and DS have prepared the manuscript after thoroughly reviewing available latest evidence on the topic and both have read and approved the final manuscript.

\section{Ethics approval and consent to participate N/A}

\section{Consent for publication}

N/A

\section{Competing interests}

Dr. SR and Dr. DS have no competing interests.

\section{Publisher's Note}

Springer Nature remains neutral with regard to jurisdictional claims in published maps and institutional affiliations.

\section{Author details}

'Gynaecworld, Kwality House, 1st Floor, Kemps Corner, Mumbai, India. ${ }^{2}$ Scientific Director \& Fertility Expert, Gynaecworld, Kwality House, 1st Floor, Kemps Corner, Mumbai, India.

Received: 5 July 2017 Accepted: 25 September 2017

Published online: 11 October 2017

\section{References}

1. Butts S, Reithman H, Ratchffe S, Shaunik A, Coutafaris C, Barnhart K Correlation of telomere length \& telomerase activity with occult ovarian insufficiency. J Clin Endo. Metabolism. 2009:94:4835-43.

2. Toner JP, Philput CB, Jones GS, Muasher SJ. Basal follicle-stimulating hormone level is a better predictor of in vitro fertilization performance than age. Fertil Steril. 1991;55:784-91.

3. Szafarowska M, Jerzak M. Ovarian aging \& infertility. Ginekol Pol. 2013 Apr; 84(4):298-304

4. Yun BH, Kim G, Park SH, EB NOC, Seo SK, et al. vitro fertilization outcome in women with diminished ovarian reserve. Obstet Gynecol Sci. 2017;60(1):4652

5. Cooper AR, Baker VL, Sterling EW, et al. The time is now for a new approach to primary ovarian insufficiency. Fertil Steril. 2012;95:1890-7.

6. Ferraretti AP, Marca L, Fauser BC, Tarlatzis B, Wargund G, et al. ESHRE consensus on the definition of 'poor response' to ovarian stimulation for in vitro fertilization: the Bologna Criteria. Hum Reprod. 2011;26:1616-24.

7. Roustan A, Perrin J, Debals-Gonthier M, Paulmyer-Lacroix O, Agostini A, Courbiere B. Surgical diminished ovarian reserve after endometrioma cystectomy versus idiopathic DOR: comparison of IVF outcome. Hum Reprod. 2015:30:840-7.

8. Devine K, Mumfard SL, Wu M, De cherney AH, Hill MJ, Propst A. Diminished ovarian reserve in the United States assisted reproduction technology population: diagnostic trends among 181,536 cycles from the Society for Assisted Reproductive Technology Clinic Outcomes Reporting System. Fertil Steril. 2015 Sep:104(3):612-9.

9. Broer S, Mol B, Hendricks D, Broekmans F. The role of anti mullerian hormone in prediction of outcome after IVF: comparison with antral follicle count. Fertil Steril. 2009;91:705-13.

10. Gleicher N, Weghofer A, Barad DH. AMH defines, independent of age, low versus good live-birth chances in women with severely diminished ovarian reserve. Fertil Steril. 2010:94:2824-7.

11. Hendricks D, Mol B, Bancsi L, teVelde E, Broekmans F. Antral follicle count in the prediction of poor ovarian reserve and pregnancy after in-vitro fertilization: a meta-analysis \& comparison with basal FSH level. Fertil Steril. 2005:83:291-301.

12. Beerendonk CC, Braat DD. Present and future options for the preservation of fertility in female adolescents with cancer. Endocr Dev. 2005;8:166-75

13. Nelson LM. Primary ovarian insufficiency. New Eng J of Med. 2009;360:60614.

14. Morita Y, Tilly JL. Oocyte apoptosis: like sand through an hour glass. Dev Biol. 1999:213:1-17.
15. Practice Committee of the American Society for Reproductive Medicine Testing and interpreting measures of ovarian reserve: a committee opinion. Fertil Steril 2015;103:e9-17.

16. Tal R, Seifer DB. Ovarian reserve testing: a user's guide. Expert Review. Am J Obstet Gynecol. 2017 Feb 21;

17. Kwee J, Schats R, McDowell's J, Lambalk CB, Schoemaker J. Intercycle variability of ovarian reserve tests: results of a prospective randomized study. Hum Reprod. 2004;19:590-5.

18. Jayprakasan K, Campbell B, Hopkisson J, Clewes J, Johnson I, Raine Fenning $\mathrm{N}$. Establishing the intercycle variablility of three dimensional ultrasonographic predictors of ovarian reserve. Fertil Steril. 2008;90:2126-32.

19. Esposito MA, Coutifaris C, Barnhart KTA. moderately elevated day 3 FSH concentration has limited predictive value, especially in younger women. Hum Reprod. 2002;17:118-23.

20. Broekmans FJ, Kwee J, Hendricks DJ, Mol BW, Lambalk CBA. systematic review of tests predicting ovarian reserve and IVF outcome. Hum Reprod Update. 2006;12:685-718.

21. Smotrich DB, Widra EA, Gindoff PR, Levy MJ, Hall JL, Stillman RJ. Prognostic value of day 3 estradiol in IVF. Fertil Steril. 1995:64:1136-40.

22. La Marca A, Sighinolfi G, Raeli D, et al. Antimullerian hormone (AMH) as a predictor marker in assisted reproductive technology (ART). Hum Reprod Update. 2010;16:113-30.

23. Garcia- Velaso JA, Moreno L, Pacheco A, et al. The aromatase inhibitor letrozole increases the concentration of intraovarian androgens and improves IVF outcome in low responder patients: a pilot study. Fertil Steril. 2005:84:82-7.

24. Grossman MP, Nakajima ST, Fallat ME, Siow Y. Mullerian inhibiting substance inhibits cytochrome P450 aromatase activity in human granulose lutein cell culture. Fertil Steril. 2008;89(Suppl):1364-70.

25. Durlinger AL, Grujiters MJ, Kramer p, et al. AMH attenuates the effects of FSH on follicle development in the mouse ovary. Endocrinlogy. 2001;142: 4891-9.

26. Fanchin R, Bouyer J. High reproducibility of serum AMH measurements suggests a multi-staged follicular secretion and strengthens its role in the assessment of ovarian follicular status. Hum Reprod. 2005;20:923-7.

27. Hekenkamp WJ, CW L, Themmen AP, De long FH, Velde T, Broekmans FJ. AMH levels in spontaneous menstrual cycle do not show substantial fluctuation. J Clin Endocrinol Metab. 2006:91:4057-63.

28. Marca L, Stabile G, Arlenisco AC, Volpe A. Serum AMH throughout the human menstrual cycle. Hum Reprod. 2006;21:3103-7.

29. Tsepelidis S, Devreker F, Demeestere I, Flahaut A, Gervy C, Englert Y. Stable serum levels of $\mathrm{AMH}$ during the menstrual cycle: a prospective study in normo-ovulatory women. Hum Reprod. 2007;22:1837-40.

30. Van Disseldrop J, Lambalk CB, Kwee J, et al. Comparison of inter and intracycle variability of $\mathrm{AMH}$ and antral follicle counts. Hum Reprod. 2010:25:221-7.

31. Overbeek A, Broekmans FJ, Hehenkamp WJ, et al. Intra-cycle fluctuations of $\mathrm{AMH}$ in a normal women with a regular cycle: a re-analysis. Reprod BioMed Online. 2012;24:664-9.

32. Sowers $M, M c D o n n e l l ~ D, ~ G a s t ~ K$, et al. AMH \& Inhibin B variability during normal menstrual cycles. Fertil Steril. 2010;94:1482-6.

33. Hansen KR, Hodnett GM, Knowlton N, Craig LB. Correlation of ovarian reserve tests with histologically determined primordial follicle number. Fertil Steril. 2010:95:170-5.

34. Kelsey TW, Anderson RA, Wright $P$, et al. Data-driven assessment of the human ovarian reserve. Mol Hum Reprod. 2012;18:79-87.

35. Freeman EW, Sammel MD, Lin H, Boorman DW, Gracia CR. Contribution of the rate of change of antimullerian hormone in estimating time to menopause for late reproductive age women. Fertil Steril. 2012 Nov;98(5):1254-9.

36. Freeman EW, Sammel MD, Lin H, Boorman DW, Gracia CR. Antimullerian hormone as a predictor of time to menopause in late reproductive age women. J Clin Metab. 2012;97:1673-80.

37. Niodromoti S, Kelsey TW, Wu O, Anderson RA, Nelson SM. The predictive accuracy of antimullerian hormone for live birth after assisted conception: a systematic review \& meta-analysis of the literature. Hum Reprod Update. 2014;20:560-70

38. Tal R, Tal O, Serfer BJ, Seifer DB. Antimullerain hormone as predictor of implantation and clinical pregnancy after assisted conception: a systematic review and meta-analysis. Fertil Steril. 2015:103:119-30.

39. Nelson SM, La Marca A. The journey from the old to the new AMH assay: How to avoid getting lost in the values. Reprod BioMed Online. 2011:23: 411-20. 
40. Nelson SM, Pastuszek E, Klos G, et al. Two new automated, compared with two enzyme -linked immunosorbent, AMH assay. Fertil Steril. 2015;104: $1016-21$.

41. Van Helden J, Weiskenchen R. Performance of two new fully automated AMH immunoassays compared with the clinical standard assay. Hum Reprod. 2015:30:1918-26.

42. Lin YH, Chu WC, Ch W, Tzung CR, Cs Hs4, Mi H. Antimullerian hormone and PCOS. Fertil Steril. 2011:96:230-5.

43. Bentzen JG, Forman JL, Pinborg A, et al. Ovarian reserve parameters. A comparison between users and non-users of hormonal contraception. Reprod BioMed Online. 2012;25:612-9.

44. Kallio S, P J, FRuokonen A, Vaskivuo T, Pulfonen T, Tapainen JS. AMH levels decrease in women using combined contraception independently of administration route. Fertil Steril. 2013;99:1305-10.

45. Butts SF, Seuifer DB. Racial and ethnic differences in reproductive potential across the life cycle. Fertil Steril. 2010;93:681-90.

46. Waylen $\mathrm{AL}$, Jones $\mathrm{GL}$, Ledger WL. Effect of cigarette smoking upon reproductive hormones in women iof reproductive age: a retrospective analysis. Reprod BioMed Online. 2010;20:861-5.

47. Dennis NA, Houghton LA, Jones GT, Van Ry AM, Morgan K, Mc Lenna IS. The level of serum anti-mullerian hormone correlates with vitamin D status in men and women but not in boys. J Clin Endocrinol Metab. 2012;97:2450-5.

48. Broekmans FJ, de Zeigler D, Howmes CM, Gongeon A, Trew G, Olivennes F. The antral follicle count, practical recommendation for better standardization. Fertil Steril. 2010;94:1044-51.

49. Kaur M, Arora M. Diminished Ovarian Reserve, Causes, Assessment and Management. Int J of Infertility and Fetal Med. 2013:4(2):45-55.

50. Gabreel A, Maheshwara A, Bhattacharya S, Johnson NP. Ultrasound tests of ovarian reserve: A systematic review of accuracy in predicting fertility outcomes. Hum Fertil. 2009;12(2):95-106.

51. Leader B, Hegde A, Baca Q, et al. High frequency of discordance between anti-mullerian hormone and follicle stimulating hormone levels in serum from estradiol-confirmed days 2 to 4 of the menstrual cycle from 5,354 women in US fertility centers. Fertil Steril. 2012;98:1037-42.

52. Toner JP, Seifee DB. Why we may abandon basal FSH testing: A sea change in determining ovarian reserve using AMH. Fertil Steril. 2013;99:1825-30.

53. La Marca A, Sighinoffi G, Giulene S, et al. Normal serum concentration of AMH in women with regular menstrual cycles. Reprod BioMed Online. 2010;21:463-9.

54. La Marca A, Papaleo E, Grisendi V, Agento C, Giulene S, Volpe A. Development of a normogram based on markers of ovarian reserve for the individualization of the FSH starting dose in in vitro fertilization cycles. BJOG. 2012 Sep;119(10):1171-9.

55. Pigny $P$, Jonard $S$, Robert $Y$, Dewailly $D$, Serum $A M H$. as a surrogate for antral follicle count for definit of the PCOS. Clin Endocrinol Metab. 2006;91: 941-95

56. Yussef MA, Vander Veen F, AL-Inany HG, et al. Gonadotropin-releasing hormone agonist versus HCG for oocyte triggering in antagonist-assisted reproductive technology. Cochrane Database Syst Rev. 2014 Oct 31;10: CD008046.

57. Le Velde ER, Pearson PL. The variability of female reproductive aging. Human. Reprod Update. 2002;8:141-54.

58. Schmidt L, Sobotka T, Bentzen JG, Nyboe Anderson A. ESHRE Reproduction and Society Task Force. Demographic and medical consequences of the postponement of parenthood. Hum Reprod Update. 2012;18:29-43.

59. Azhar E, Seifer DB, Melzer K, Ahmed A, Weedon J, Minkoff H. Knowledge of ovarian reserve and reproductive choices. J Assist Reprod Genet. 2015;32: 409-15.

60. Tremellen K, Savulescu J. Ovarian reserve screening: A scientific and ethical analysis. Hum Reprod. 2014;29:2604-14.

61. Ouddendijk JF, Yarde F, Eijkemans MJ, Broekmans FJ, Broer SJ. The poor responder in IVF: Is the prognosis always poor? : A systematic review. Hum Reprod Update. 2012 Jan - Feb;18(1):1-11.

62. Atabekoglu C. Climacteric 2012; 15(4): 393-397 Tasken S, Kahraman K, Gemici A, et al. The effect of total abdominal hysterectomy on seven antimullerian hormone levels: A pilot study.

63. Farquhar CM, Sadler L, Harvey SA, Stewart AW. The association of hysterectomy and menopause : A prospective cohort study. BJOG. 2005 July;112(7):956-62.

64. Raffi F, Metwally M, Amer S. The impact of excision of ovarian endometrioma on ovarian reserve : A systematic review and meta-analysis. J Clin Endocrinol Metab. 2012 Sep;97(9):3146-54.
65. Amer SA, El Shamy TT, James C, Yosef AH, Mohamed AA. The impact of laparoscopic ovarian drilling on $\mathrm{AMH}$ and ovarian reserve: a meta - analysis. Reproduction. 2017 Apr 18;

66. Mohamed AA, Yosef AH, James C, Al-Hussaini TK, Bedaimj, SAKS A. Ovarian Reserve after Salpingectomy : A systematic review. Acta Obstet Gynecol Scand. 2017 Mar 17;

67. MCKinlay SM, Biffano NL, McKinlay JB. Smoking and age of menopause in women. Ann Intern Med. 1985;103(3):350-6.

68. Peres J. New hope for young breast cancer patients. JNCIO. 2014;106(9)

69. Gotlieb WH, Beiner ME, Shalmon B, Korach Y, Segal Y, et al. Outcome of fertility - sparing treatment with progestin in young patients with endometrial cancer. Obstet Gynecol. 2003 Oct;102(4):718-25.

70. Lallemant C, Vassard D, Nyboe Anderson A, et al. Medical and social egg freezing: internet-based survey of knowledge ans attitudes among women in Denmark and UK. Acta Obstet Gynecol Scand. 2016 Dec;95(12):1402-10.

71. Papathanasiou A, Searle BJ, King NM, Bhattacharya S. Trends in 'poor responder' research: Lessons learned from RCTs in assisted conception. Hum Reprod Update. 2016 Apr; 22 (3).

72. Pandian Z, Mc Tavish AR, Aucoyy L, Hamilton MP, Bhattacharya S. Interventions for 'poor responders' to controlled ovarian hyperstimulation $(\mathrm{COH})$ in IVF. Cochrane Database Systematic Review. 2010 Jan 20;1

73. Ben Rafael Z, Benadiva CA, Ausmanas M, et al. Dose of human menopausal gonadotropin influences the outcome of an IVF. Fertil Steril. 1987:48:964-8.

74. Alviggic C, Anderson CY, Buehler K, Corforti A, De Placido G, Esteves SC, Fischer R, Galliano D, Polyzoz NP, Sunkara SK, Ubaildi FM, Humaisan P. a new more detailed stratification of low responders to ovarian stimulation: From a poor ovarian response to a low prognosis concept. Fertil Steril. 2016 Jun;105(6):1452-3.

75. Van Hooff MH, Alberda AT, Hussman GJ, Zeilmaker GH, Lerrentveld RA. Doubling the dose of human menopausal gonadotropin in course of IVF treatment cycle in poor responder : A randomized study. Hum Reprod. 1993;8:369-73.

76. Friedler S, Meltzer S, San - Ryss B, Robinson J, Lazer T, Libaty G. An upper limit of gonadotropin dose in patient undergoing Art should be advocated. Gynecol Endocrinol 2016 Dec; 32 (12): 965-969.

77. Macklon NS, Fauser BC. Progress in ovarian stimulation. Ann Endocrinol (Paris). 1999 Jul;60(2):137-42.

78. Macklon NS, Fauser BC. Impact of ovarian hyperstimulation on the luteal phase. J Reprod Fertil Suppl. 2000;55:101-8.

79. Merviel P, Cabry-Goubet R, Lourdel E, Devaux A, et al. Comparative prospective study of 2 ovarian stimulation protocols in poor responders: effect on implantation rate and ongoing pregnancy. Reprod Health. 2015 May 30;12:52.

80. Xiao J, Chang S, Cheu S. The effectiveness of gonadotropin - releasing hormone antagonist in poor ovarian responders undergoing in in vitro fertilization: A systematic review and meta - analysis. Fertil Steril. 2013 Dec; 100(6):1594-601.

81. Leondires MP, Escalpes M, Segaus JH, Scott RT Jr, Muller BT. Microdose follicular phase gonadotropin releasing hormone agonist impared with luteal phase agonist for ovarian stimulation in in vitro fertilization. Fertil Steril 1999; 72: 1018-1023.

82. Song Y, Li Z, We X, Wang X, Xicu J, Nang B. Effectiveness of the antagonist or letrozole protocol for treating poor responders undergoing in vitro fertilization or intra Cytoplasmic sperm injection : A systematic review and meta-analysis. Gynecol Endocrinol. 2014 May;30(5):330-4.

83. Surrey ES, Bower J, Hill DM, Ramsey J, Surrey MW. Clinical and endocrine effects of a microdose $\mathrm{GnRH}$ agonist flare regimen administered to poor responders who are undergoing in vitro fertilization. Fertil Steril. 1999;69(3): 419-24.

84. Toth TL, Awwad JT, Veeck LL, Jones HW Jr, Muasher SJ. Suppression and flare regimens of gonadotropin-releasing hormone agonist. Use in women with different basal gonadotropin values in an in vitro fertilization program J Reprod Med 1996 May:41(5):321-326.

85. Dirnfeld M, Fruchter D, Yshar A, et al. Cessation of GnRH - a upon down regulation versus conventional long $\mathrm{GnRH}$ - a - protocol in poor responders undergoing IVF. Fertile Steril 1999; 72 (NO): 406-11.

86. Garcia-velasco JA, Isaza V, Requena A. High doses of gonadotrophins combined with stop versus non - stop protocol of $\mathrm{GnRH}$ - analogue administration in low responder IVF patients: A prospective, randomized controlled trial. Hum Reprod. 2000;15(11):2292-6.

87. Kyrou D, Kolibeanakis EM, Vanetis EG, Papanekolaon G, et al. How to improve the probability of pregnancy in poor responders undergoing IVF: A systematic review and meta - analysis. Fertil Steril. 2009;91(3):749-66. 
88. Polyzos NP, Devos M, Humaidan P, et al. Corifollitropin alfa followed by $r$ FSH in a GnRH antagonist protocol for poor ovarian responder patients: An observational pilot study. Fertil Steril. 2013;99(22):422-6.

89. Polyzos NP, Devos M, Corona R, et al. Addition of highly purified HMG after corifollitropin alfa in antagonist treated poor ovarian responders : A pilot study. Hum Reprod. 2013;28(5):1254-60.

90. Yang R, Li H, Li R, Liu P, Qiau JA. comparison among different methods of letrozole combined with gonadotropins in an antagonist protocol and high dose gonadotropin ovarian stimulation antagonist protocol in poor ovarian responders undergoing IVF. Arch Gynecol Obstet. 2016 Nov;294(5):1091-7.

91. Lazer T, Dar S, Shlush E, Al Kudmani BS, Quach K, et al. Comparison of IVF outcomes between minimal stimulation and High - dose stimulation for patients with poor ovarian reserve. Int J Reprod Med. 2014;2014:581451.

92. Youssef MA, van Wely M, Al-Inany H, Madani T, Jahangiri $N$, et al. A mild ovarian stimulation strategy in women with poor ovarian reserve undergoing IVF; A multicenter randomized non-inferiority trial. Hum Reprod. 2017 Jan;32(1):112-8

93. Song D, Shi Y, Zhong Y, Meng Q, Hon S, Li H. efficiency of mild ovarian stimulation with clomiphene on poor ovarian responders during IVF / ICSI procedures : A meta - analysis. End J Obstet Gynecol Reprod Biol. 2016 Sep; 204:36-43.

94. Oktem M, Guler I Endern M, et al, Comparison of the effectiveness of Clomiphene citrate versus letrozole in mild IVF in poor prognosis subfertile women with failed IVF cycles. Int J fertile Steril . 2015 Oct - Dec; 9(3): 285291.

95. Nagels HE, Reshworth JR, Serestatides CS, Kroon B. Androgens (DHEA or T) for women undergoing assisted reproduction. Cochrane Database Systematic Review. 2015 Nov 26; (11): CD 009749.

96. Reynolds KA, Omurtag KR, Jimenez PT, Rhee JS, Tuuli MG, Jungheim ES. Cycle cancellation and pregnancy after luteal estradiol priming in women defined as poor responders : A systematic review and meta - analysis. Hum Reprod. 2013;28(11):2981-9.

97. Helher SG, Zelenik AJ, Ron GT. Independence of steroidogenic capacity and luteinizing hormone receptor induction on developing granulosa cells. Endocrinology. 1978;102:937-46.

98. Hsu CJ, Hammond JM. Concomitant effects of growth hormone on secretion of insulin like growth factor I and progesterone by cultural porcine granulose cells. Endocrinology. 1987;121(4):1343-8.

99. Li XL, Li W, Lei F, Huang XM, et al. The influence of different growth hormone addition protocols to poor ovarian responders on clinical outcomes in controlled ovary stimulation cycles. Medicine (Baltimore). 2017; 96(12):1-8.

100. Kolibianakis EM, Venetis CA, Diedrich K, Tarlotzis C, Griesinger G. Addition of growth hormone to gonadotropin in ovarian stimulation of poor responders treated by IVF : A systematic review and meta-analysis. Hum Reprod Update. 2009;15(6):613-22.

101. Woo MM, Tai CJ. kang SK, Nathiwani A et al. Direct action of melatonin in human granulose - luteal cells. J Clin Endocrinol Metab. 2001;86:4789-97.

102. Jahromi BN, Sadeghi S, Alipour S, Parsanehzad ME, Alamdarloo SM. Effect of Melatonin on the outcome of ART cycles in women with DOR : A double blinded randomized clinical trial. Iran J Med Sci. 2017 Jan;42(1):73-8.

103. Pellicer A, Baester J, Serrano MD, et al. Aetiological factors involved in the low response to gonadotropins in infertile women with normal basal serum follicle - stimulating hormone levels. Hum Reprod. 1994;9(5):806-12.

104. Battaglia C, Gena zzani AD, Regnani G, et al. Perifollicular Doppler flow and follicular fluid vascular, endothelial growth factor concentration in poor responders. Fertil Steril. 2000;74(4):809-12.

105. Gelbaya TA, Kyrgeou M, Li TC, Stern C, Nardo LG. Low - dose aspirin for iVF: A systematic review and meta-analysis. Human. Reprod Update. 2007;13(4): 357-64.

106. Cobo A, Garrido N, Crespo J, Jox A, Pellicer A. Accumulation of oocytes : A new strategy for managing low - responder patients. Reprod BioMed Online. 2012 Apr;24(4):424-32.

107. Gougeon Al. neogenesis in the adult ovary a realistic paradigm? Gynecol Obstet Fertil. 2010 Jun;38(6):398-401.

108. Yang DZ, Yang W, Li Y, He Z. progress in understanding human ovarian folliculogenesis and its implications in assisted reproduction. J Assist Reprod Genet. 2013 Feb;30(2):213-9.

109. Kansal Kalra S, Ratcliffe S, Gracia CR, Martino L, et al. Randomized controlled pilot trial of luteal phase recombinant FSH stimulation in poor responders. Reprod BioMed Online. 2008 Dec;17(6):745-50.
110. Kuang Y, Chen Q, Hong Q, et al. Double stimulation during the follicular and luteal phases of poor responders in IVF/ICSI programmes. Reprod BioMed Online. 2014 Dec;29(6):684-91.

111. Zhang Q, Guo XM, Li Y. Implantation rates subsequent to the transfer of embryos produced at different phases during double stimulation of poor ovarian responders. Reprod Fertil Dev. 2016 May 11;

112. Zou K, Yuan Z, Yang Z, et al. Production of offspring from a germline stem cell line derived from neonatal ovaries. Nat Cell Biol. 2009 May;11(5):631-6.

113. Virant-klun I, Zech $N$, Rozman $P$, et al. Putative stem cells with an embryonic character isolated from the ovarian surface epithelium of women with no naturally present follicles and oocytes. Differentiation. 2008 Oct;76(8):843-56.

114. Virant-klun I, Rozman P, Cvieticamin B, et al. Parthogenetic embryo - like structures in the human ovarian surface epithelium cell culture in post menopausal women with no naturally present follicles and oocytes. Stem cell dev. 2009 Jan-Feb;18(1):137-49.

115. Shah D, Rasool S, Noronha C. Gynaecworld unpublished data.

\section{Submit your next manuscript to BioMed Central and we will help you at every step:}

- We accept pre-submission inquiries

- Our selector tool helps you to find the most relevant journal

- We provide round the clock customer support

- Convenient online submission

- Thorough peer review

- Inclusion in PubMed and all major indexing services

- Maximum visibility for your research

Submit your manuscript at www.biomedcentral.com/submit
) Biomed Central 